duced a rigid body nutation. In fact, calculations show that the Nix Olympica mass, for example, is likely to give a rigid body nutation of only $0.04^{\circ}$. Thus even the effect of the four largest shield areas will be at least two orders of magnitude smaller than the polar wandering implied by the quasi-circular polar features. But is Mars a totally rigid planet? The fact that the observed value of the primary coefficient of the low order harmonics of the Martian gravity field is close to that expected from a rotating planet in hydrostatic equilibrium suggests that, although Mars has a rigid surface, its interior may be hot enough to support viscous motion. In this case, the nutation could be much higher than that predicted on the basis of a rigid body. Moreover, the presence of viscous motion itself implies mass movements much greater than those represented solely by the exposed shields. The existence of what amounts to mantle convection would be consistent with polar wandering but, as in the case of the Earth, would not be sufficient to prove it.

A third feature of Mars relevant to the polar wandering argument is that the low degree spherical harmonic coefficients (non-hydrostatic) of the gravity field are relatively larger than those of the Earth. On Mars they also correlate significantly with surface topography and geology, whereas they do not do so on Earth. Thus, for example, the apex of the equatorial bulge for both the gravity field and the planetary surface correlate well with the location of the four large shield volcanoes. This phenomenon could be taken to support the connexion between large-scale volcanism and deep-seated density differences which would be expected at the start of large-scale mantle convection, although, perhaps unfortunately, an interpretation could also be made in terms of a totally rigid Mars. To this extent, the gravity data are equivocal.

Nevertheless, at least three different aspects of Mars are at least consistent with polar wandering. But if polar wandering has taken place, or is taking place, what is its rate? The equatorial shield areas contain few impact craters, and so they are probably no older than a few hundred million years. This gives a rough idea of the time scale involved. The polar features may, however, give a more accurate result. Assuming, as Leighton and Murray (Science, 153, 136 ; 1966) did, that each lamella of the laminated terrain represents a 50,000 -year climatic period, the clearly distinguishable polar plates may represent about $5 \times 10^{7} \mathrm{yr}$, and the more eroded plates offset from the present axis by up to $20^{\circ}$ may represent about $10^{8} \mathrm{yr}$. On this basis, the polar wandering rate would be about $5 \times 10^{-9} \mathrm{rad}_{\mathrm{yr}^{-1}}$ or about 20 arc min per million years.
NUCLEAR STRUCTURE

\section{Testing New Models}

from a Correspondent

THE study of nuclear physics has long since fissioned into nuclear structure and high energy physics, a division which is formally recognized in conferences such as that held in Liverpool from March 28 to 30 under the auspices of the Institute of Physics. (In turn there has appeared to be subdivision within nuclear structure physics too, but the boundaries seem less clear.)

At the Liverpool conference Dr R. Chapman (University of Manchester) surveyed the evidence for a dramatic departure from the rotational model sequence of energy levels which occurs at high angular momentum in heavy nuclei. This phenomenon is now ascribed to a phase change in the nucleon motion from that of a superfluid to that of a near rigid rotor. Thus from failures of simple models much is learnt about the physics of the nucleus. The same general point was mentioned by $\mathrm{Dr}$ R. R. Whitehead (University of Glasgow) in the very different context of large-basis shell model calculations for light nuclei. By ingenious computational techniques and the use of large computers it is now possible to make traditional shell model calculations for nuclei in the $2 \mathrm{~s}-1 \mathrm{~d}$ shell without resorting to the usual pruning of smaller wavefunction components. Dr Whitehead argued that whereas it was possible to contrive agreement between model and experiment by using effective nuclear forces to cover up for a truncated calculation, a better idea of the real nuclear forces would be learnt from the success or failure of the fuller calculations.

Although recognizing the importance of these mammoth calculations, many physicists--and especially experimenters - feel the need for a complementary simple model whereby they may grasp some meaning from the various data over a range of nuclei. Dr P. W. M. Glaudemans (University of Utrecht) described such a model for $s-d$ shell nuclei using the so-called surface-delta interaction. Although this schematic model is nominally of the shell model type, the effective interaction used enables it to reproduce some of the observed strong electromagnetic transition strengths which betray the presence of collective nuclear motion.

The experimental evidence for such collective motion in $s-d$ shell nuclei has grown rapidly in the past few years.

\title{
Explaining Electric Detonation of Explosives
}

Although it is well known that solid explosives can be detonated by electric fields and electric discharges, it is not clear just what mechanisms are at play in the process. Some have suggested that, in the case of azides like $\mathrm{Cu}\left(\mathrm{N}_{3}\right)_{2}$, the explosion can be traced back to a breakdown of electrical resistance caused by the injection of holes from the anode or of electrons from the cathode. Another question that arises is whether mechanical shock or thermal shock arising from the electrical breakdown is responsible for the triggering of the explosion.

In next Monday's Nature Physical Science (April 16) Chaudhri describes a study by means of high speed photography of the early stages of this kind of detonation. The figure shows a sequence of photographs taken at a rate of 5 million frames a second of the stages in the decomposition of a single crystal of silver azide $\left(\mathrm{AgN}_{3}\right)$. A negative pulse of amplitude $10 \mathrm{kV}$ and rise time about $0.5 \mu \mathrm{s}$ was applied between frames 2 and 3 , and it reached a maximum between frames 4 and 6 . Two dark expanding regions $S$ and $S_{1}$ are in evidence in frame 6 , and, significantly, they are well away from the electrodes. The later frames suggest that the dark regions arose from shock waves. Chaudhri says, however, that it is not clear whether the shock waves he observed are the result of electrical breakdown or the initiation of fast decomposition at these points.

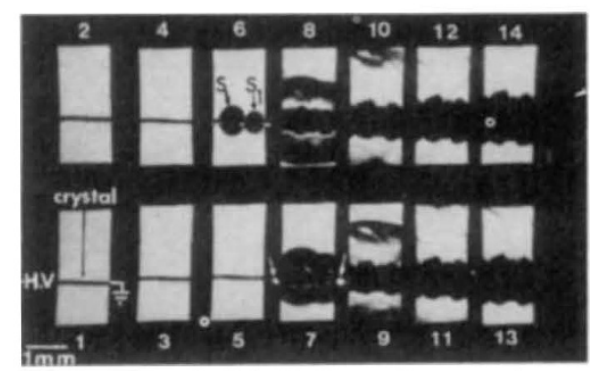

The electrical initiation of a reaction in a crystal of $\mathrm{AgN}_{3}$. The electrodes are steel plates. The single crystal is $\sim 100 \mu \mathrm{m}$ in diameter, and the whole arrangement is situated in a container filled with oil.

Another sequence of photographs, this time taken at a rate of 10 million frames a second, reveals an initial dark spot in the crystal near, but not at, the anode. It would seem that the propagation speed of the reaction was some 1,750 $\mathrm{m} \mathrm{s} \mathrm{s}^{-1}$, about 70 per cent greater than the normal propagation speed. Chaudhri concludes that the reaction does not start because of impact ionization and decomposition by electrons injected from the cathode, but he says that more work will have to be done to pin the mechanism down. 